\title{
Contrast Negation and the Importance of the Eye Region for Holistic Representations of Facial Identity
}

\author{
Mladen Sormaz, Timothy J. Andrews, and Andrew W. Young \\ University of York
}

\begin{abstract}
Reversing the luminance values of a face (contrast negation) is known to disrupt recognition. However, the effects of contrast negation are attenuated in chimeric images, in which the eye region is returned to positive contrast (S. Gilad, M. Meng, \& P. Sinha, 2009, Role of ordinal contrast relationships in face encoding, Proceedings of the National Academy of Sciences, USA, Vol. 106, pp. 5353-5358). Here, we probe further the importance of the eye region for the representation of facial identity. In the first experiment, we asked to what extent the chimeric benefit is specific to the eye region. Our results showed a benefit for including a positive eye region in a contrast negated face, whereas chimeric faces in which only the forehead, nose, or mouth regions were returned to positive contrast did not significantly improve recognition. In Experiment 2, we confirmed that the presence of positive contrast eyes alone does not account for the improved recognition of chimeric face images. Rather, it is the integration of information from the positive contrast eye region and the surrounding negative contrast face that is essential for the chimeric benefit. In Experiment 3, we demonstrated that the chimeric benefit is dependent on a holistic representation of the face. Finally, in Experiment 4, we showed that the positive contrast eye region needs to match the identity of the contrast negated part of the image for the chimera benefit to occur. Together, these results show the importance of the eye region for holistic representations of facial identity.
\end{abstract}

Keywords: face recognition, eyes, photo negation, contrast chimera, contrast

Reversing the luminance values of an image (contrast negation) disrupts the recognition of familiar faces (Galper, 1970; Galper \& Hochberg, 1971; Phillips, 1972; Johnston, Hill, \& Carmen, 1992). The effect of contrast negation provides an interesting counterexample to the typical resistance of familiar face recognition to other types of image degradation (Bruce \& Young, 2012), and contrast negation appears to disrupt face recognition to a greater degree than recognition of other types of visual stimuli (Vuong, Peissig, Harrison, \& Tarr, 2005; Nederhouser, Yue, Mangini, \& Biederman, 2007). The effect of contrast negation is easily seen (by those old enough to remember chemical photography) in the difficulty of recognizing faces from photographic negatives. However, the negative of a normal color image reverses both its luminance and its hue. A seminal study by Kemp, Pike, White, and Musselman (1996) demonstrated that face images with negated luminance but normal hue saw a reduction in recognition accuracy, whereas faces with negated hue and normal luminance showed no recognition accuracy decrease. It is therefore clear that information critical to face recognition is somehow carried through luminance values.

The explanation for why contrast reversal has such a profound effect on face recognition has been dominated by two different

This article was published Online First May 13, 2013.

Mladen Sormaz, Timothy J. Andrews, and Andrew W. Young, Department of Psychology, University of York, UK.

We thank Mel Irwin and Emily Lloyd, whose undergraduate project provided pilot assessments for some of the experiments reported here, though in the event none of their data were used.

Correspondence concerning this article should be addressed to Mladen Sormaz, Department of Psychology, University of York, Heslington, York YO10 5DD, UK. E-mail: ms930@york.ac.uk ideas. The first is Kemp et al.'s (1996) suggestion that the difficulty in recognizing contrast negated faces is due to the reversal of three-dimensional (3D) shape from shading cues. Their underlying premise is that disrupting depth cues through contrast reversal interferes with the use of information about 3D shape. However, more recent studies have tended to downplay the potential role of $3 \mathrm{D}$ information in face recognition and place more emphasis on the importance of surface pigmentation (Bruce \& Young, 2012). For example Liu, Collin, and Chaudhuri (2000) found that recognition was poor for faces with intact 3D information but missing surface pigmentation. In line with such findings, Bruce and Langton (1994) developed the second main approach to the impact of contrast negation by suggesting that it exerts its disruptive influence because it reverses the brightness of important pigmented regions of the face, so that light regions of skin become dark, dark hair becomes light, and so forth. Recent work expanding on this hypothesis has been carried out by Russell, Sinha, Biederman, and Nederhouser (2006), who propose that much of the contrast negation effect results from negating pigmentation cues that carry substantial information about facial identity. This has been elaborated by Sinha, Russell, and their colleagues (Sinha, Balas, Ostrovsky, \& Russell, 2006; Russell \& Sinha, 2007) into a more general claim that because pigmentation carries much information about facial identity, any manipulation (e.g., contrast negation) that disrupts pigmentation rather than shape will have deleterious effects on recognition. Consistent with this hypothesis, further work by Santos and Young (2008) shows that contrast negation effects are not only confined to identity judgments but also impact on a range of social inferences made to faces in which pigmentation cues are likely to play a substantial role. Importantly, too, a study by White (2001) showed a degree of independence of 
contrast negation effects on facial identity and expression. White found that the perception of facial expression was much less affected by contrast negation than perception of identity. Since contrast negation does not affect the locations of regions of rapid change in luminance that define the shapes of facial features critical to the perception of expression, this dissociation is in line with the suggestion that contrast negation effects disrupt facial characteristics heavily dependent on pigmentation cues (i.e., identity) rather than shape (i.e., expression).

Recently, however, a dramatic finding by Gilad, Meng, and Sinha (2009) has added to the debate about how and why contrast reversal disrupts recognition of identity by demonstrating that neither the pigmentation nor the shape from shading accounts given previously can be entirely correct. Gilad and colleagues (2009) created "contrast chimeras" of famous individuals consisting of contrast negated faces in which the eye regions were left in positive contrast. Strikingly, recognition accuracy for these contrast chimeras was around $90 \%$ of the recognition rate for normal face photographs. This result is remarkable as it shows that merely restoring the correct contrast polarity to a small region around the eyes is sufficient to eliminate much of the classic contrast negation effect on face identification. Yet the major proportion of a contrast chimera image still has reversed luminance values and therefore gives incorrect shape from shading and incorrect pigmentation cues. Traditional approaches leave it unclear why restoring correct luminance values to only a small part of the image should have such a profound effect.

Gilad et al. (2009) suggested that this "contrast chimera effect" arises because the eye region plays a critical role in creating a visual representation of a face and a contrast chimera face image maintains the natural ordinal contrast relationships around the eye region. More specifically, they point out that the ordinal relations between relatively lower luminance levels in the eye region and higher luminance values of the surrounding cheeks, nose and forehead remain constant across nearly all natural lighting conditions and viewpoints in which we encounter faces. Because these relationships are generally so stable, Gilad and colleagues suggested that the eyes provide a reliable feature around which to create face representations. Negating the contrast of the whole face reverses the natural ordinal contrast relationships between the eyes and surrounding face, disrupting the critical role of this region. Contrast chimeras however maintain the normal lower luminance levels of the eye region and higher luminance values of the surrounding face regions, therefore maintaining the correct ordinal contrast relationships from which an overall representation of the face can be created. This hypothesis offers a potentially important new perspective that regards ordinal contrast polarity relationships in the eye region as central to facial identity representations.

The aim of the present study is to use the chimeric effect to probe further how the eye region is involved in the perception of facial identity. In the first experiment, we asked whether the chimeric benefit is specific to the eye region or whether it can be found with chimeras in which the forehead, nose and mouth region are returned to contrast positive. In the second experiment, we investigated whether the eye region alone is sufficient or whether integration of information from the positive contrast eye region and the surrounding negative contrast face is essential for the chimeric benefit. In the third experiment, we tested whether the chimeric benefit is dependent on a holistic representation of faces.
In the final experiment, we asked how precisely the information from the positive contrast eye region needs to match that from the contrast negated part of the face for the chimera benefit to occur.

\section{Experiment 1}

A first step toward understanding the contrast chimera effect is to identify the key facial region involved. Although Gilad et al.'s (2009) account focused on the eyes, they noted that any beneficial effect of creating contrast chimeras based on the mouth region did not reach statistical significance, but did not report testing the effect of putting any other facial regions into photo positive. Because recent work by Ohayon, Friewald, and Tsao (2012) has shown increased neural responses when macaques were exposed to noneye regions from the face that had consistent ordinal contrast relationships (e.g., forehead-nose), Experiment 1 investigated whether contrast chimeras involving other regions of the face might also improve recognition compared to full contrast negated stimuli. Specifically, we compared recognition rates for contrast chimeras involving the forehead, eyes, nose, or mouth regions. These regions were chosen in part based on the work of Dakin and Watt (2009), which suggested the nose and mouth regions as well as the eyes contain potentially informative horizontally oriented spatial frequencies. The forehead was chosen as a control region unlikely to contain critical information, so that it could be used as a baseline from which to measure whether simply revealing any region of the face in positive contrast might improve recognition accuracy.

In Gilad et al.'s (2009) study they created each of their chimeric stimuli by carefully blending the positive contrast eye region with the rest of the contrast negated image. This way of creating contrast chimeras makes it less obvious how the image has been changed and allows the region of change to be tightly circumscribed. However, because we wanted to arrive at a manipulation that would be equivalent across different face regions, we opted instead to use a simpler technique of using a constant-shaped rectangular frame to delineate the contrast positive region, with no blending of the borders of this rectangular frame or other artistic effects. This allowed us to move the frame up or down within an image to bring into positive contrast equivalently shaped regions highlighting the forehead, eyes, nose, or mouth.

\section{Method}

Stimuli. Face images were produced using Adobe Photoshop version 5. Photographs of 32 (16 male, 16 female) famous and highly recognizable individuals (actors, politicians, and other famous people) were used. Care was taken to ensure that each image was of a similar size, pose and image resolution, and that the faces were of people well-known to most undergraduate students in the U.K. (established by pilot screening). All images used were as close as possible to full face views. All face images in result figures are from the White House website (www.whitehouse.gov) and depict current USA President Barack Obama. Images were greyscaled and cropped to only show the face (including hair), and superimposed on a uniform gray background. Faces were presented in a pseudorandom order in a 32-page booklet, with one face per page at the center of an A4 sheet of paper. When viewed from around $30 \mathrm{~cm}$ away, each image subtended on average 13 degrees horizontally and 16 degrees vertically. 
Each of the 32 greyscale face images was converted into a contrast negated (greyscale photo negative) version, and for each of these contrast negated images chimeric variants were produced by putting a constantly sized and shaped rectangular region around the forehead, eyes, nose, or mouth into photo positive. Examples of these manipulations are shown in Figure 1.

Participants. Forty participants (mean age: 22 years, 20 females), took part in Experiment 1. All participants were from a Western cultural background and had normal or corrected to normal vision. The study was approved and conducted following the guidelines of the Ethics committee at the University of York Psychology Department. Participants were paid or took part for course credit.

Design and procedure. An independent measures design was used, with each participant allocated to one of five image manipulation conditions (eight participants per group). Each participant saw a pseudorandomized sequence of faces in a booklet corresponding to their allotted image manipulation condition. They were asked to identify each face in an answer booklet. Correct recognition occurred when the full name of the face, an alias or an identifier unique to an individual (e.g., David Cameron could be identified as "Current prime minister of the U.K.") were provided.

The five experimental conditions were as follows:

(i) A full contrast negative face.

(ii) Forehead chimera: negative contrast face except for a rectangular positive contrast forehead region. The positive contrast forehead region did not overlap the space covered in any other chimera conditions and was the same size as that used to create the eye chimera.

(iii) Eye chimera: each image seen by participants in this group was contrast negated except for a rectangular positive contrast eye

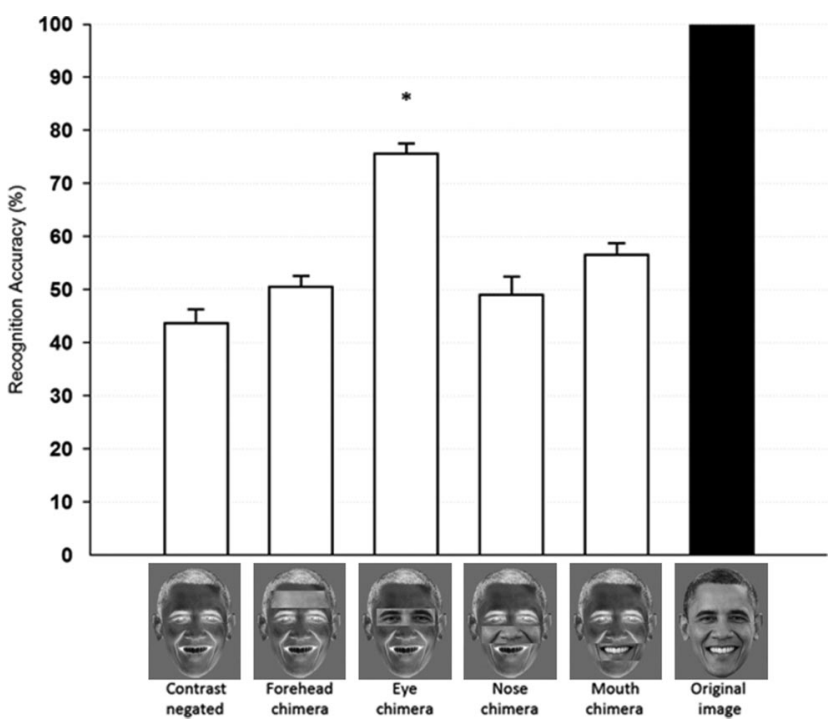

Figure 1. Histogram displaying recognition accuracy in each condition of Experiment 1, with standard error. Performance of the experimental conditions is conditionalized on recognition of the original image photographs. Asterisk marks condition with superior recognition rate in comparison to contrast negated images and all other chimera manipulations. All face images are from the White House website (www.whitehouse.gov) and depict current USA President Barack Obama. region. The eye regions were demarcated to contain at least the whole eye and eyebrows of each individual.

(iv) Nose chimera: each image seen by participants in this group was contrast negated except for a positive contrast nose region. Again, the positive contrast nose region did not overlap the space covered in any other chimera conditions and was the same size as that used to create the eye chimera.

(v) Mouth chimera: each image seen by participants in this group was contrast negated except for a positive contrast mouth region. The positive mouth region did not overlap the space covered in any other chimera conditions and was the same size as the eye chimera.

After completing their designated image manipulation condition, all participants were given a booklet of the same 32 faces in normal greyscale image form presented in a new pseudorandom order, and were again asked to identify each face. This was used as an "original image" control condition to determine which faces were known to each participant.

\section{Results}

Accuracy for each condition was calculated as a proportion of the faces recognized in the original image control condition (the normal greyscale images). In this way, faces that were not recognized in the control condition (i.e., faces that were unknown to participants) were eliminated from the analysis. A similar procedure was used by Gilad et al. (2009) and gives rise to recognition accuracy in the original image control condition of $100 \%$.

Percentage correct recognition accuracies in each condition are shown in Figure 1. Statistical analyses were conducted both by participants and by items.

For the analysis by participants, the percent correct scores for the five image manipulation conditions were arcsine converted and subjected to a one-way between-groups ANOVA. Because of the use of an arcsine transform, mean accuracy results are given below in radians as well as the more easily interpreted percentages. The ANOVA indicated a significant effect of the image manipulation condition on recognition accuracy with a large effect size $\left(F_{4,35}=\right.$ 6.93, $\left.p<.001, n^{2}=.44\right)$. A Tukey's HSD post hoc test was carried out with a corrected alpha value $(\mathrm{a}=.05$, critical arcsine value $=.25$ radians) to compare performance between conditions. This showed that performance in the eye chimera condition $(M=$ $75.7 \%, S D=10.7$, mean radians $=.87$ ) was significantly higher than for the contrast negated $(M=43.8 \%, S D=14.2$, radians $=$ $.46)$, forehead chimera $(M=50.6 \%, S D=11.2$, radians $=.53)$, nose chimera (accuracy $=49 \%, S D=19.9$, radians $=.53$ ) and mouth chimera (accuracy $=56.6 \%, S D=12.7$, radians $=.61$ ) conditions. There were no significant differences between the four remaining conditions (contrast negated, forehead chimera, nose chimera, and mouth chimera).

To test whether the effects revealed from the analysis by participants generalized across stimulus items (i.e., across familiar faces), and to complement the limited power of the betweenparticipants design used, we also carried out an items analysis of our data. Percentage correct scores for each face in the five image manipulation conditions were arcsine converted and subjected to a one-way between-groups ANOVA. This items analysis revealed a pattern of results that matched the main analysis by participants. There was a significant effect of the image manipulation condition 
on recognition accuracy with a medium effect size $\left(F_{4,155}=5.66\right.$, $\left.p<.001, n^{2}=.14\right)$. A Tukey's HSD post hoc test $(\mathrm{a}=.05)$ showed that performance in the eye chimera condition was significantly higher than for the contrast negated, forehead chimera, nose chimera and mouth chimera conditions. There were no significant differences between the four remaining conditions (contrast negated, forehead chimera, nose chimera, and mouth chimera).

\section{Discussion}

Our results show that the chimeric benefit is maximal for the eye region. No significant improvements in face recognition were apparent for chimeras in which the forehead, nose, or mouth region were in contrast positive compared to full negative contrast faces. That said, the overall recognition accuracy for eye region contrast chimeras $(76 \%)$ did not seem as high as that in Gilad and colleagues' study (91\%). This may be due to differences in recognizability across the 32 famous faces we used compared to the eight faces used by Gilad et al. (2009), or it might result from our less "natural" rendering of the contrast positive region by simply "letterboxing" it. This was done to make the area of the image returned to photo positive consistent across the chimeric conditions, but it introduces irrelevant lines into each image that define the border of the rectangular area, and adding irrelevant lines to an image of a face can affect its recognizability (Ellis, Davies \& Shepherd, 1978). The key point, though, is that in spite of this slightly reduced accuracy for our eye region contrast chimeras compared to that observed by Gilad et al. (2009), there was none the less a substantial benefit to recognition from returning the eye region of a contrast negated face back into contrast positive.

The finding that this contrast chimera benefit only existed in the eye chimera condition suggests that Gilad et al. (2009) correctly pinpointed the region of the face where ordinal contrast relationships appear to be most important in identity recognition. This is interesting as recent work by Dakin and Watt (2009) indicates that faces carry potentially useful high spatial frequency information in the horizontal plane especially around the eyes, nose, and mouth. Consequently, creating chimeras with positive contrast in the nose and mouth regions, which contain the aforementioned horizontal high spatial frequency information, might also have improved recognition. While inspection of Figure 1 does suggest that a study with greater statistical power might reveal some benefit for the mouth chimeras, any such effect is much less pronounced than for the eyes.

\section{Experiment 2}

Having established from Experiment 1 that the eye region offers the greatest recognition benefit for contrast chimeric images, Experiment 2 was carried out to test how the different parts of the eye chimeric image might be used by the observer in creating this contrast chimera benefit. In Gilad et al.'s (2009) paradigm, the contrast chimeric benefit is primarily measured by the difference in performance between a contrast chimera (the "normal eye chimera" in Figure 2) and a standard greyscale negative (shown as "contrast negated" in Figure 2). Logically, the only difference between these images is that the eye region of the normal eye chimera is contrast positive. Gilad et al.'s (2009) interpretation of

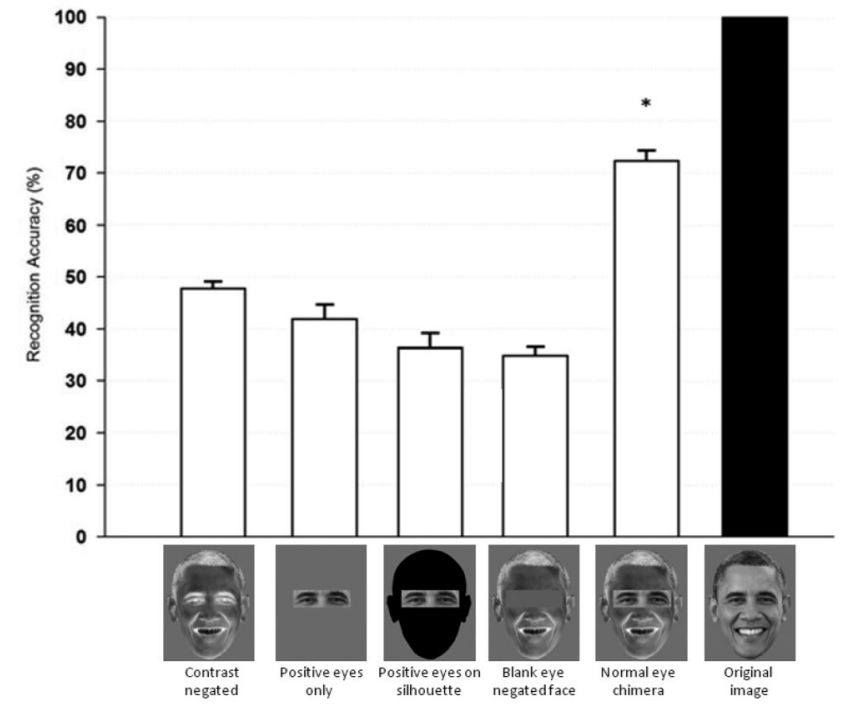

Figure 2. Histogram displaying recognition accuracy in each condition of Experiment 2, with standard error. Asterisk marks the condition with a higher recognition rate than all other experimental conditions. All face images are from the White House website (www.whitehouse.gov) and depict current USA President Barack Obama.

the benefit of adding this contrast positive eye region was that it allowed information from the contrast negated region to be used to assist in identifying the individual. However, there is a variety of other possible reasons, of which we sought to test three:

- First, there might be enough information in the positive eye region to allow recognition based on using that region alone, and ignoring the rest of the chimeric stimulus. For this reason, we included an "eyes only" condition.

- Second, the combination of a correct eye region might be used together with a fairly general cue from the negated region, such as overall shape. For this reason, we included an "eyes on silhouette" condition in which the contrast positive eye region was placed on a correct face outline for the person. Gilad et al. (2009) had also used this condition, but we thought it important to replicate it here because our participants' overall performance with the contrast chimeras seemed lower than that found by Gilad et al. (see discussion of Experiment 1).

- Third, the benefit might be less to do with the presence of a contrast positive eye region than with the removal of the misleading contrast negative eye region. For this reason, we included a "blank eye negated" condition to see how well the rest of a contrast negated face could be recognized if the misleading eye region was removed.

\section{Method}

Stimuli. The same greyscale images of 32 famous individuals (16 male, 16 female) used in Experiment 1were used to create stimuli for Experiment 2.

Participants. Forty new participants (mean age: 21 years, 20 females) participated in Experiment 2. All participants met the same demographic requirements as those in Experiment 1. 
Design and procedure. An independent measures design was used with each participant allocated to one of five image manipulation conditions (eight participants per group):

(i) Contrast negated: each image seen by participants in this condition was a full contrast negated image.

(ii) Positive eyes only: each image seen by participants in this condition was a positive contrast eye region, the same size and shape as that in the normal eye chimera condition, superimposed on a homogeneous gray background.

(iii) Positive eyes on face silhouette: each image seen by participants in this condition involved a positive contrast eye region of the same size and shape as that in the normal eye chimera condition, superimposed in the correct position on a head shape silhouette.

(iv) Blank eye negated face: each image seen by participants in this condition was contrast negated but with a homogeneous gray eye region that was the same size and the same average luminance as the eye region in the normal eye chimera condition.

(v) Normal eye chimera: each image seen by participants in this group was manipulated in the same way as those from the eye chimera condition in Experiment 1.

All other aspects of design and procedure were as for Experiment 1. As in Experiment 1 an "original image" control condition was seen by all participants after their allocated image manipulation condition, to determine which faces each participant could recognize from a normal greyscale image.

\section{Results}

Percentage correct recognition accuracy scores were collated following the same method as in Experiment 1 and can be seen in Figure 2. For statistical analysis, the results were again analyzed by participants and by items.

For the analysis by participants, percent correct scores for the five image manipulation conditions were arcsine converted and subjected to a one-way between-groups ANOVA. This indicated a significant effect of the image manipulation condition on recognition accuracy with a large effect size $\left(F_{4,35}=10.09, p<.001\right.$, $\left.n^{2}=.52\right)$. A Tukey's HSD post hoc test (corrected alpha value a $=$ .05 , critical Tukey's value $=.26$ ) was used to compare performance between conditions. Results indicated that recognition accuracy in the normal eye chimera condition $(M=72.4 \%, S D=$ 13.2 , radians $=.83$ ) was significantly higher than contrast negated $(M=47.8 \%, S D=9.7$, radians $=.50)$, positive eyes on silhouette $(M=36.4 \%, S D=18.6$, radians $=.38)$, blank eye negated $(M=$ $34.9 \%, S D=11.3$, radians $=.36)$ and eyes only $(M=41.9 \%$, $S D=18.1$, radians $=.44)$ conditions. Comparisons between the remaining conditions (contrast negated, positive eyes on silhouette, blank eye negated, and eyes only) showed no significant differences.

As for Experiment 1, in Experiment 2 we carried out an items analysis on our data. The percent correct scores for the five image manipulation conditions were arcsine converted and subjected to a between-groups ANOVA. However, as the assumption of homogeneity of variance was violated, we used Welch's $F$ to adjust the degrees of freedom and the $F$ ratio. This items analysis revealed a pattern of results that matched the main analysis by participants. Welch's $F$ indicated a significant effect of the image manipulation condition on recognition accuracy with a large effect size
$\left(F_{4,76.2}=8.05, p<.001, \mathrm{w}^{2}=.41\right)$. A Games-Howell post hoc test was used to compare performance between conditions. Results indicated that recognition accuracy in the normal eye chimera condition was significantly higher than for contrast negated ( $p=$ $.01)$, positive eyes on silhouette $(p=.001)$, blank eye negated $(p=.001)$ and eyes only $(p=.001)$ conditions. Comparisons between the remaining conditions (contrast negated, positive eyes on silhouette, blank eye negated, and eyes only) showed no significant differences (all $p$ values greater than .05).

\section{Discussion}

The recognition accuracy data indicate that only the normal eye chimera condition led to a boost in performance, with all other image manipulations showing no difference from the baseline contrast negated condition. These findings are important as they show that specific components of the contrast chimeras cannot support good recognition rates alone. For example, the positive eye region alone is not particularly well recognized, and this is the case even when it is combined with the face's silhouette. Neither does the contrast negated region seem to contain useful cues in the absence of positive eyes. The benefit created by the positive eye region of the contrast chimeras therefore arises from some form of interaction between information from the positive eye region and the contrast negated remainder of the image. Experiment 3 investigated the nature of this interaction.

A minor point is that the recognition of "positive eyes on face silhouette" did not differ significantly from the contrast negated condition, whereas Gilad et al. (2009) found that their equivalent condition was actually more detrimental to recognition than their contrast negated condition. This may reflect the fact that the technique we used to create the contrast chimeras results in somewhat larger positive eye regions, therefore revealing more of the face and possibly giving a slightly stronger cue to identity from the eye region itself than the method used by Gilad and colleagues.

\section{Experiment 3}

Experiment 3 investigated how the positive eye region interacts with the contrast negated part of the image to boost recognition of contrast chimeras. Specifically, we looked at whether the chimera benefit results from holistic perception of the face-like chimeric image. To achieve this, we tested whether it would be possible to create the contrast chimera effect by presenting the components of a contrast chimera in a configuration that was not face-like.

The concepts of "configuration" or "configural processing" are widely used in face perception research, but unfortunately can carry different meanings (Bruce \& Young, 2012). Maurer, Le Grand, and Mondloch (2002) distinguish three importantly different senses in which such terms are often used. These are the first-order configuration of eyes above nose above mouth shared by all faces, the second-order configuration involving the precise spacings of these features unique to an individual, and third the concept that the face is processed "holistically" rather than as a collection of parts. For Experiment 3, we created a separate eyes and head chimera condition in which the eye region was placed alongside the rest of the face. This arrangement violates the firstorder configural properties as defined by Maurer et al. (2002), and violations of the first-order configuration (such as isolating or 
misaligning facial features) are known to severely disrupt holistic processing (Young, Hellawell, \& Hay, 1987; Tanaka \& Farah, 1993; Maurer et al., 2002; Bruce \& Young, 2012).

We therefore applied the same criterion to the contrast chimera effect, asking whether it, too, would be disrupted by violating first-order configural properties by spatially separating the parts of the chimera. This was done by placing the positive eye region and outer negated face region of a chimera alongside each other (see Figure 3). Logically, these separate eye and head chimeras present the same information as a normal eye contrast chimera. Psychologically, though, the information is not in face-like form. If the contrast chimera effect involves the creation of a holistic percept, the recognition accuracy for a normal contrast chimera in a facelike first-order configuration should be better than the recognition accuracy for its spatially separated components. If no difference in recognition is seen between these two conditions, it would imply that the chimeric benefit results from a more "piecemeal" processing of the constituent facial features (Carey \& Diamond, 1977).

\section{Method}

Stimuli. The 32 normal eye chimera greyscale images from Experiments 1 and 2 were used, and spatially rearranged versions of each were created.

Participants. Sixteen new participants (Mean age $=21$ years, 8 females) participated in Experiment 3. All participants fulfilled the same demographic requirements as those in Experiments 1 and 2 .

Design and procedure. An independent measures design was used, with each participant allocated to one of two image manipulation conditions (eight participants per group):

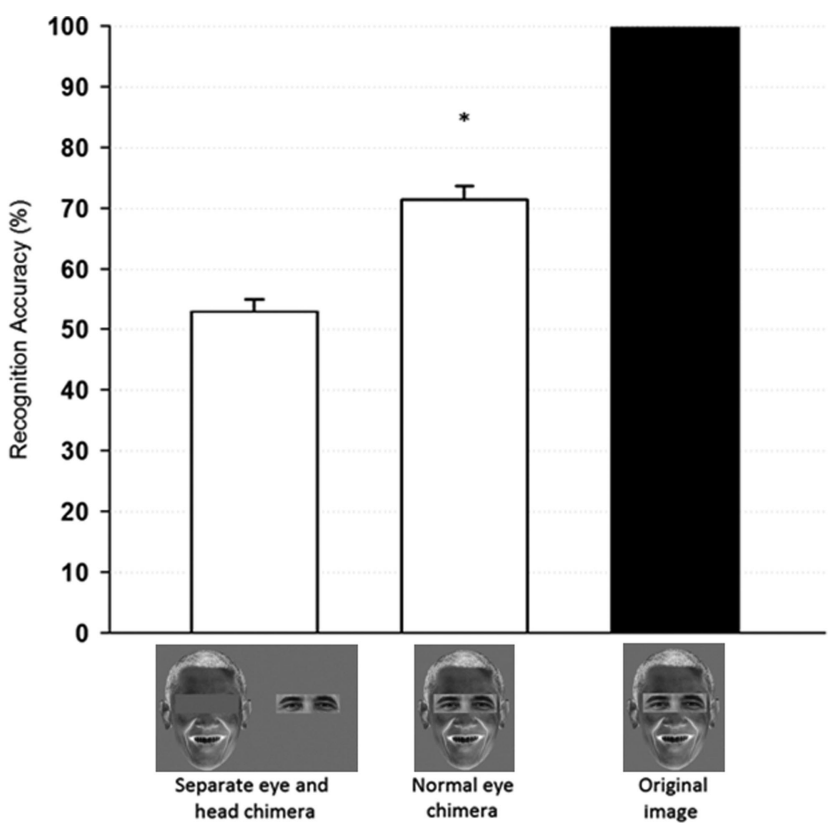

Figure 3. Histogram displaying recognition accuracy in each condition of Experiment 3, with standard error. Asterisk marks normal eye chimera showing a significantly higher recognition rate than the separate eye and head chimera. All face images are from the White House website (www .whitehouse.gov) and depict current USA President Barack Obama. (i) Separate eye and head chimera: each face seen by participants in this condition consisted of a contrast negated face with a homogeneous gray eye region presented adjacent to a positive contrast eye region from the same image. The distance between the eye region alone and the blank eye region was that of the distance from the ear to the edge of the positive eye region in the individual. Hence the stimuli comprised the same components as a standard contrast chimera, but placed alongside each other.

(ii) Normal eye chimera: the same stimuli as the "eye chimera" condition in Experiment 1 and the "normal eye chimera" condition in Experiment 2.

All other aspects of design and procedure were as for Experiments 1 and 2. Again an "original image" control condition was seen by all participants after their allocated image manipulation condition, to determine which faces each participant could recognize from a normal greyscale image.

\section{Results}

Recognition accuracy was calculated using the same method as in Experiments 1 and 2 and can be seen in Figure 3. The percent correct recognition accuracies in each condition were arcsine transformed for statistical analysis. For the analysis by participants, an independent $t$ test was used to probe the difference between the separate eyes and head chimera and normal eye chimera conditions. The $t$ test showed that there was a significant difference in recognition accuracy between the normal eye chimera $(M=71.46 \%, S D=13.3$, radians $=.95)$ and the separate eye and head chimera $(M=52.9 \%, S D=11.7$, radians $=.60)$ conditions, $t_{(14)}=2.81, p=.014, r^{2}=.36$.

An items analysis using an independent $t$ test revealed a pattern of results that matched the main analysis by participants. The $t$ test showed that there was a significant difference in recognition accuracy between the normal eye chimera and the separate eye and head chimera conditions, $t_{(62)}=2.61, p=.011, r^{2}=.10$.

\section{Discussion}

The contrast chimera benefit was greater when the positive contrast eye region was presented within a face-like configuration, demonstrating that it involves some form of holistic face processing. Having established that holistic processing of the contrast positive and contrast negated parts of the chimera takes place, we turned our attention to how precise this holistic processing might be, addressing Gilad et al.'s (2009) hypothesis of the importance of ordinal contrast relationships in the eye region.

\section{Experiment 4}

As Experiments 1-3 all pointed to the importance of interaction between information from the positive and negative parts of the chimera in the eye region, we sought to clarify how this happens by looking more closely at the role played by ordinal contrast relationships. As already noted, Gilad et al.'s (2009) account of the contrast chimera benefit is that the pattern of ordinal contrast relations around the eyes forms such a stable feature of the faces we see that the brain incorporates this stability into its perceptual representation of faces, leading to a severe decrement in recognition when the critical eye region is contrast negated. This account 
is couched in terms of a general property of faces, raising the question of whether any transform that can restore a normal set of ordinal contrast relationships to the eye region of a contrast negated face might make it more recognizable. Note that Gilad et al.'s (2009) account does not predict this would be the case-it only emphasizes the stability of ordinal contrast relationships as inherent to the importance of the eye region. We sought to test how precisely the eye region must match the negated part of a contrast chimera for a benefit in recognition to accrue.

In a standard contrast chimera, as used here in Experiments 1-3, the chimera is created from a single face photograph. Because the positive eye region and the contrast negated part of the chimera both come from the same photograph, they necessarily match each other in two important ways-in identity (the parts come from images of the same person, even though one part is negated) and in the pattern of illumination (the parts come from images that are equivalently lit, even though one part is negated). In Experiment 4, we sought to unpack these influences as far as practicable, by including conditions that took the positive eye region of the chimera from a different photograph of the same person as that used for the contrast negated part (creating a difference in pattern of illumination between the parts of the chimera) or that took the positive eye region of the chimera from a photograph of a different person from that used for the contrast negated part (creating a difference in identity and in pattern of illumination between the parts of the chimera). In both of these conditions any overall ordinal contrast relations that are common to all face images will remain unchanged.

\section{Method}

Stimuli. The contrast negated and contrast chimera images of the 32 famous individuals used in Experiments 1-3 were used as the "contrast negated" and "normal eye chimera" conditions for Experiment 4.

Two additional chimera conditions were also created. The first was created by using the positive eye regions from a second photograph of each of the faces of the 32 famous people in the stimulus set and combining these with the contrast negated images from the original set of photographs to create "same eye identity but different image" chimeras in which the positive and negative parts came from different images of the same person. The second was done by using the positive eye regions from photographs of the faces of 32 famous people who were not in the original stimulus set and combining these with the contrast negated images from the original set to create "different eye identity" chimeras.

A pilot study was used to check that the two images of each face used to create the "same eye identity but different image" chimeras were clearly perceptibly different in the eye region. In this pilot, 10 participants (Mean age $=23,5$ females) completed a simple "same/different" task where they were required to judge whether two sequentially presented pairs of eyes were from the same or different images. In each case the identity of the eyes remained unchanged across images; the only possible change was that the two images were or were not identical. Participants had to distinguish whether eye pairs were same or different image pairs for each of the 32 famous faces used. Results indicated that the eyes used to create the "same eye identity but different image" chimera stimuli could be easily distinguished from the "normal eye chi- mera" eyes based on similar performance in both the same eye and different eye conditions (Different accuracy $=97 \%, S D=7.9$; same accuracy $=96 \%, S D=6.6$ ).

Participants. Thirty-two participants (Mean age $=21$ years, 16 females) participated in Experiment 4. All participants fulfilled the same demographic requirements as those in Experiments 1, 2, and 3 .

Design and procedure. An independent measures design was used with each participant allocated to one of four image manipulation conditions (eight participants per group):

(i) Contrast negated: each image seen by participants in this condition was a full contrast negated image, as in Experiments 1 and 2.

(ii) Different eye identity (ID) chimera: each image seen by participants in this condition involved a contrast negated version of one of the individuals from the standard image set combined with a positive contrast eye region of the same size as that used for the normal eye chimera condition, but with this eye region belonging to one of four famous individuals (Orlando Bloom for Caucasian males, Sienna Miller for Caucasian females, Tyra Banks for nonCaucasian females, Tiger Woods for non-Caucasian males) of the same sex and ethnicity.

(iii) Same eye identity (ID) different image chimera: each image seen by participants in this condition involved a contrast negated version of one of the individuals from the standard image set combined with a positive contrast eye region of the same size as that used for the normal eye chimera condition, but with this eye region taken from a different photograph of the same individual.

(iv) Normal eye chimera: the images seen by participants in this group were of the same individuals manipulated the same way as for the "eye chimera" condition in Experiment 1 and the "normal eye chimera" condition in Experiments 2 and 3.

All other aspects of design and procedure were as for Experiments 1 to 3 , with an "original image" control condition at the end of the session, to determine which of the 32 faces in the main set of stimuli could be recognized by each participant from a normal greyscale image.

\section{Results}

Recognition accuracies calculated using the same method as in Experiments 1-3 can be seen in Figure 4. Note that in the different eye ID chimera condition there are a variety of possible responses that might occur, based on identifying the image in terms of the positive eye region, in terms of the negated rest of the face, recognizing it as an entirely unrelated person, or complete inability to recognize it as anyone familiar. In practice, the majority of responses involved either recognition based on the negated part $(38 \%)$ or a complete inability to recognize it as anyone familiar (40\%), whereas incorrect identification where an answer was produced $(20 \%)$ and identification based on the eye region alone $(2 \%)$ was relatively rare. In light of this, we decided to allow responses based on either the negated part of the image or on the positive eye region as "correct." This is a valid tactic because either of the eye or the negated face regions contains one identity and, therefore, depending on which facial areas are used by the viewer for identification, either answer can be viewed as based on information present in the stimulus. 


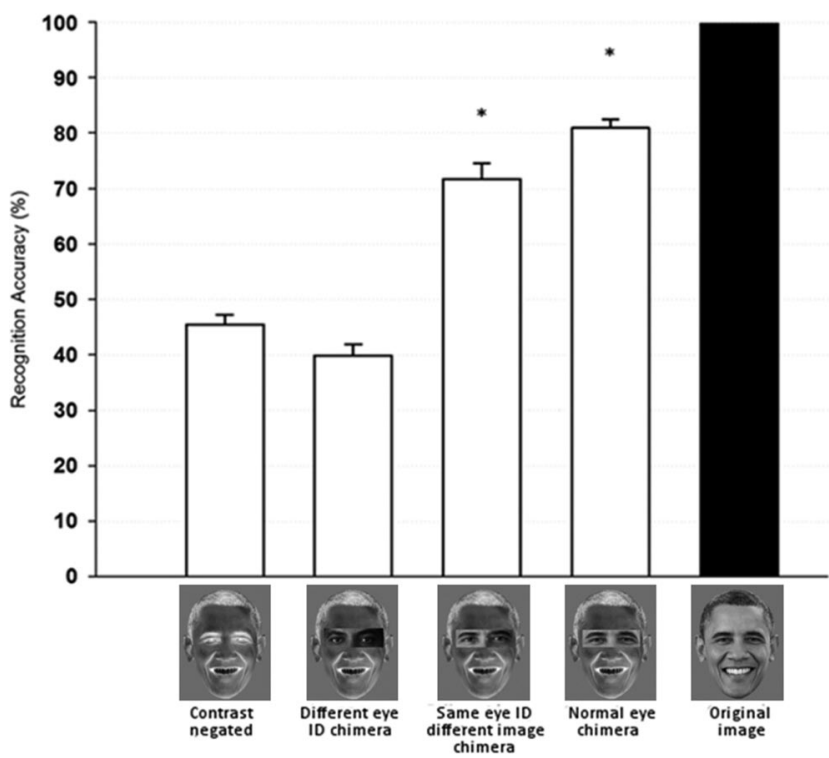

Figure 4. Histogram displaying recognition accuracy in each condition of Experiment 4, with standard error. Asterisks denote conditions with significantly greater recognition accuracy than contrast negated and different eye ID chimera conditions. Performance for the two asterisked conditions does not differ significantly from each other. All face images are from the White House website (www.whitehouse.gov) and depict current USA President Barack Obama.

For the analysis by participants, percent correct recognition accuracies in each condition were arcsine transformed (results also stated in arcsine radians) and submitted to a one-way betweengroups ANOVA. This indicated a significant effect of the image manipulation condition on recognition accuracy with a large effect size $\left(F_{4,35}=15.67, p<.001, n^{2}=.63\right)$. A Tukey's HSD post hoc test (corrected alpha value a $=.05$, critical Tukey's value $=.28$ ) was used to compare performance between conditions. This showed that the mean performance in the normal eye chimera condition $(M=81 \%, S D=8.4$, radians $=.96)$ was significantly higher than for the contrast negated $(M=45.5 \%, S D=11.1$, radians $=.48)$ and different eye ID chimera $(M=39.9 \%, S D=$ 11.1 , radians $=.44$ ) conditions. However, performance in the normal eye chimera condition did not differ significantly from the same eye ID different image chimera condition $(M=71.7 \%$, $S D=16.8$, radians $=.84$ ). Instead, the same eye ID different image chimera condition also showed significantly higher recognition accuracy than the different eye ID chimera and contrast negated conditions. The comparison between the different eye ID chimera and contrast negated conditions showed no significant difference.

A separate items analysis revealed a pattern of results that matched the main analysis by participants. There was a significant effect of the image manipulation condition on recognition accuracy with a medium effect size $\left(F_{3,127}=15.91, p<.001\right.$, $n^{2}=.28$ ). A Tukey's HSD post hoc test (corrected alpha value $\mathrm{a}=.05$ ) showed that the mean score in the normal eye chimera condition was significantly higher than for the contrast negated and different eye ID chimera conditions. However, performance in the normal eye chimera condition did not differ significantly from the same eye ID different image chimera condition. Instead, the same eye ID different image chimera condition also showed significantly higher recognition accuracy than the different eye ID chimera and contrast negated conditions. The comparison between the different eye ID chimera and contrast negated conditions showed no significant difference.

\section{Discussion}

The results showed that contrast chimeras created from two different images of the same face (same eye ID different image chimeras) were as effective in promoting recognition as chimeras made from parts of the same photograph (normal eye chimera condition). So the contrast chimera effect shows some degree of tolerance of lighting changes. However, putting someone else's eyes into a contrast chimera (the different eye ID chimera condition) nullified the chimeric benefit and brought performance back to the level found for fully negated images.

It is of course possible that an experiment with more power might reveal a statistically reliable difference between the "same eye ID different image" chimera and the normal eye chimera conditions, but for present purposes this is not the main point, since both of these conditions led to a substantial boost in recognition compared to the different eye ID chimera condition. This clearly implies that identity information from the eye region is a critical component of the contrast chimera benefit. The mere presence of ordinal contrast relationships in the eye region that approximate those of any normal face (as in the different eye ID chimera condition) is not sufficient. If ordinal contrast relationships are the key determinant of the effect, they are sufficiently precisely specified as to be specific to the relevant identity. This suggests some level of interdependency of ordinal contrast relationships and shape cues in the eye region for representations of identity.

\section{General Discussion}

The aim of this study was to use the contrast chimera effect to explore the importance of the eye region for face recognition. The results clearly confirm Gilad et al.'s (2009) observation that restoring the correct contrast polarity to the eye region of a contrast negated face image confers a considerable benefit to recognition. However, our findings go further and demonstrate a number of important properties of this contrast chimera effect. First, the benefit to recognition of contrast negated face images from creating a contrast chimera containing a positive contrast eye region is a robust, easily replicable phenomenon. Second, the eye region does seem critical, since only contrast chimeras based around the eye region produced any statistically significant benefit for identification. Third, the contrast chimera benefit does not arise because the positive eyes are themselves intrinsically highly recognizable; it reflects a genuine interaction between the positive and negated regions of the chimera. Fourth, the contrast chimera effect reflects holistic processing of the image; the components are most effective in promoting recognition when arranged in a face-like configuration. Fifth, this holistic processing of contrast chimeras uses precise information from the eye region; there is no benefit from incorrect eyes that nonetheless preserve many of the ordinal contrast relationships typical of faces seen in everyday life. 
These characteristics of the contrast chimera effect suggest that at least three interdependent properties contribute to the role of the eye region in face recognition. First, the eye region has important contrast polarity relations; Experiments $1-4$ show how reversing these impacts on recognition (cf. Gilad et al., 2009). Second, information from the eye region is used in a precise fashion when these ordinal contrast relationships are correct (Experiment 4). Third, the eye region is combined holistically with other parts of the face (Experiment 3).

Three aspects of these findings merit more general discussion. These involve their implications for understanding normal face recognition mechanisms, why the eye region might be so important, and implications for the debate about whether faces can be considered a special class of objects.

Considering the implications for understanding normal recognition mechanisms, this has of course been why the effects of contrast negation have been considered so intriguing. The paradox of contrast negation is that, strictly speaking, all of the information is still present in the negated image, yet the visual system seems unable to use it. The striking benefit from putting a relatively small region around the eyes back into photo positive therefore has important implications, particularly since (as we have demonstrated here) the benefit accrues from the participant's now being able to use information from the contrast negated region itself. As noted in our Introduction, this does not sit easily with accounts of the impact of contrast negation based only on the reversal of shape from shading or pigmentation cues, since these accounts offer no explanation as to why information from the negated region should become usable under the particular condition that the image has a positive eye region. This doesn't eliminate any role for such cues, but at the least it implies they are only part of the story.

It is interesting that previous studies have shown that contrast negation does not itself preclude holistic face processing (Hole, George, \& Dunsmore, 1999; Taubert \& Alais, 2011). Thus, our finding that the eye region contrast chimera is processed holistically with the contrast negated part of the image must fit into this more general background. Further studies might, therefore, usefully seek to clarify how the positive eye region facilitates the usefulness of information in the negated part of the chimera, which seems to us the enigma at the core of the contrast chimera effect.

One way to approach this enigma is to think more carefully about the information in a greyscale face photograph. The image involves a complex pattern of light and shade resulting from how light falls across the 3D structure of the face and the surface pigmentation of the facial features, skin and hair. Some of this pattern is potentially informative about the face's identity, but much of it also results from identity-irrelevant lighting variations and camera characteristics. Burton, Jenkins, Hancock, and White (2005) have shown how averaging a number of different images of the same person's face can eliminate most of this irrelevant variation, leading to an averaged pigmentation pattern with good recognizability. Moreover, Burton et al. (2005) noted that these averaged pigmentation patterns are sufficiently characteristic of the face in question that most of them remain recognizable even if they are themselves morphed into an average face shape. This shows that pigmentation patterns contribute sufficiently strongly to the perception of face identity that they can override 2D shape cues involving feature positioning (the "second-order configuration").
A promising direction for future work on understanding the contrast chimera effect, therefore, seems to us to explore what aspects of this pigmentation pattern can be used from the contrast reversed part of the image; for instance, whether the information accessed from the negated region involves relatively coarse or fine spatial frequencies, and whether or not it is 2D-shape dependent. Such approaches could also be used to ask whether the benefit of a correctly rendered eye region is specific to overcoming the deleterious effect of contrast negation per se, or whether it applies equally in any circumstances that create problems for face recognition. For example the addition of visual noise or some other degradation of the image might be used to reduce recognition of a face to a level comparable to that created by contrast negation, and the benefit of restoring the eye region could then be determined. A direct comparison between the benefit of restoring the eyes in a contrast chimera and in other types of degraded image could help to establish the extent to which the contrast chimera effect reflects the more general diagnostic value of the eye region in face recognition.

Why should the eye region have such privileged status? Various lines of evidence converge on the importance of the eye region in the perception and recognition of faces (McKelvie, 1976; Haig, 1986; O’Donnell \& Bruce, 2001; Barton, Radcliffe, Cherkasova, Edelman, \& Intriligator, 2006; Peterson \& Eckstein, 2012), but the contrast chimera benefit makes the point particularly effectively. Prior to the discovery of the chimera effect evidence already existed showing a substantial effect of eye inversion on face recognition (Leder, Candrian, Huber, \& Bruce, 2001; Rakover \& Teucher, 1997). Similarly, the role of holistic processing in the contrast chimera benefit matches findings showing that prosopagnosic individuals who do not process faces holistically fail to fixate on and extract diagnostic information from the eyes when trying to recognize faces (Caldara et al., 2005; Orban de Xivry, Ramon, Lefevre, \& Rossion, 2008).

There are a number of factors that may be involved in this preeminence for the eyes. Structurally the positive contrast eye region contains a number of characteristic pigmentation variations in a small area; these include the sclera, iris and pupil within the eyeball, the border between the nose and the brow, and the eyebrows, with the latter being implicated as also contributing to familiar face recognition (Sadr, Jarudu, \& Sinha, 2003). These structural properties are what leads to the presence of substantial information from horizontal spatial frequencies in the eye region described by Dakin and Watt (2009), and they create the stable ordinal contrast relationships noted by Gilad et al. (2009), which potentially offer a useful anchor point around which to construct facial identity representations. Moreover, the eyes are, of course, also important to a wide range of social signals (Bruce \& Young, 2012), so human perceivers have many reasons for looking at this region of the face. Studies in evolutionary biology suggest that the importance of signals communicated through eye gaze is such that the human eye may have evolved a distinct pigmentation pattern that can communicate gaze direction very effectively (Kobayashi \& Kohshima, 1997).

A point that should not be neglected, though, is that the importance of the eye region isn't the only thing that needs to be explained. Both our results and those of Gilad et al. (2009) show that the recognition rate for eye region contrast chimeras is nonetheless lower than that for a full positive face. So we cannot totally 
discount the usefulness of other face areas and, in fact, Sinha (2002) suggested that important relative contrast relationships may also exist in other face regions (e.g., nose-cheek) as well as the eyes. A possibility for future studies would be to test whether chimeric stimuli involving combinations of regions provide significantly better recognition than a single region chimera alone.

Finally, the findings have implications for the debate about the status of faces as special stimuli for the visual system. This debate has been bedevilled by the different meanings and interpretations given to the word special (Hay \& Young, 1982; Ellis \& Young, 1989), but the dependence of the contrast chimera benefit on the eye region of the face and the fact that few stimuli other than faces have eyes gives it a clearly unusual status that qualifies as special within most of the definitions that have been used. Indeed a number of studies have shown that in the perception of faces is actually unlike many other classes of object in that it is differentially severely impaired by contrast negation (e.g., Subramaniam \& Biederman, 1997; Nederhouser et al., 2007).

Overall, the findings presented in this paper provide a robust replication of the contrast chimera effect first found by Gilad et al. (2009) and support the importance of contrast relationships in the eye region to face recognition. When correct contrast relationships are present, information from the eye region is used in a precise but holistic fashion.

\section{References}

Barton, J. J. S., Radcliffe, N., Cherkasova, M. V., Edelman, J., \& Intriligator, J. M. (2006). Information processing during face recognition: The effects of familiarity, inversion and morphing on scanning fixations. Perception, 35, 1089-1105. doi:10.1068/pp.5547

Bruce, V., \& Langton, S. (1994). The use of pigmentation and shading information in recognising the sex and identities of faces, Perception, 23, 803-822. doi:10.1068/pp.230803

Bruce, V., \& Young, A. (2012). Face perception. Hove, UK: Psychology Press.

Burton, A. M., Jenkins, R., Hancock, P. J. B., \& White, D. (2005). Robust representations for face recognition: The power of averages. Cognitive Psychology, 51, 256-284. doi:10.1016/j.cogpsych.2005.06.003

Caldara, R., Schyns, P., Mayer, E., Smith, M. L., Gosselin, F., \& Rossion, B. (2005). Does prosopagsnosia take the eyes out of face representations? Evidence for a defect in representing diagnostic facial information following brain damage. Journal of Cognitive Neuroscience, 17, 16521666. doi:10.1162/089892905774597254

Carey, S., \& Diamond, R. (1977). From piecemeal to configurational representations of faces. Science, 195, 312-314.

Dakin, S. C., \& Watt, R. J. (2009). Biological "bar codes" in human faces. Journal of Vision, 9(4), article 2. doi:10.1167/9.4.2

Ellis, H. D., Davies, M. D., \& Shepherd, J. W. (1978). Remembering pictures of real and 'unreal' faces: Some practical and theoretical considerations. British Journal of Psychology, 69, 467-474. doi:10.1111/j .2044-8295.1978.tb02123.x

Ellis, H. D., \& Young, A. W. (1989). Are faces special? In A. W. Young \& H. D. Ellis (Eds.), Handbook of research on face processing (pp. 1-26). Amsterdam, The Nethelands: North Holland.

Galper, R. E. (1970). Recognition of faces in photographic negative. Psychonomic Science, 19, 207-208.

Galper, R. E., \& Hochberg, J. (1971). Recognition memory for photographs of faces. The American Journal of Psychology, 84, 351-354. doi: $10.2307 / 1420466$
Gilad, S., Meng, M., \& Sinha, P. (2009). Role of ordinal contrast relationships in face encoding. Proceedings of the National Academy of Sciences, USA, 106, 5353-5358. doi:10.1073/pnas.0812396106

Haig, N. D. (1986). Investigating face recognition with an image processing computer. In H. D. Ellis, M. A. Jeeves, F. Newcombe \& A. Young (Eds.), Aspects of face processing (pp. 410-425). Dordrecht, The Netherlands: Martinus Nijhoff.

Hay, D. C., \& Young, A. W. (1982). The human face. In A. W. Ellis (Ed.), Normality and pathology in cognitive functions (pp. 173-202). London, UK: Academic Press.

Hole, G. J., George, P. A., \& Dunsmore, V. (1999). Evidence for holistic processing of faces viewed as photographic negatives. Perception, 28 , 341-359. doi:10.1068/p2622

Johnston, A., Hill, H., \& Carmen, N. (1992). Recognising faces: Effects of lighting direction, inversion, and brightness reversal. Perception, 21, 365-375. doi:10.1068/p210365

Kemp, R., Pike, G., White, P., \& Musselman, A. (1996). Perception and recognition of normal and negative faces: The role of shape from shading and pigmentation cues. Perception, 25, 37-52. doi:10.1068/ p250037

Kobayashi, H., \& Kohshima, S. (1997). Unique morphology of the human eye. Nature, 387, 767-768. doi:10.1038/42842

Leder, H., Candrian, G., Huber, O., \& Bruce, V. (2001). Configural features in the context of upright and inverted faces. Perception, 30, 73-83. doi:10.1068/p2911

Liu, C. H., Collin, C. A., \& Chaudhuri, A. (2000). Does face recognition rely on encoding of 3-D surface? Examining the role of shape-fromshading and shape-from-stereo. Perception, 29, 729-743. doi:10.1068/ p3065

Maurer, D., Le Grand, R. L., \& Mondloch, C. J. (2002). The many faces of configural processing. Trends in Cognitive Sciences, 6, 255-260. doi:10.1016/S1364-6613(02)01903-4

McKelvie, S. J. (1976). The role of eyes and mouth in the memory of a face. The American Journal of Psychology, 89, 311-323. doi:10.2307/ 1421414

Nederhouser, M., Yue, X., Mangini, M. C., \& Biederman, I. (2007). The deleterious effect of contrast reversal on recognition is unique to faces, not objects. Vision Research, 47, 2134-2142. doi:10.1016/j.visres.2007 .04 .007

O'Donnell, C., \& Bruce, V. (2001). Familiarisation with faces selectively enhances sensitivity to changes made to the eyes. Perception, 30, 755764. doi:10.1068/p3027

Ohayon, S., Friewald, W. A., \& Tsao, D. Y. (2012). What makes a cell face selective? The importance of contrast. Neuron, 74, 567-581. doi: 10.1016/j.neuron.2012.03.024

Orban de Xivry, J.-J., Ramon, M., Lefevre, P., \& Rossion, B. (2008). Reduced fixation on the upper area of personally familiar faces following acquired prosopagnosia. Journal of Neuropsychology, 2, 245-268. doi:10.1348/174866407X260199

Peterson, M. F., \& Eckstein, M. P. (2012). Looking just below the eyes is optimal across face recognition tasks. Proceedings of the National Academy of Sciences, USA, 109, E3314-E3323. doi:10.1073/pnas .1214269109

Phillips, R. J. (1972). Why are faces hard to recognise in photographic negative? Perception \& Psychophysics, 12, 425-426. doi:10.3758/ BF03205854

Rakover, S. S., \& Teucher, B. (1997). Facial inversion effects: Parts and whole relationship. Attention, Perception, \& Psychophysics, 59, 752761. doi:10.3758/BF03206021

Russell, R., \& Sinha, P. (2007). Real-world face recognition: The importance of surface reflectance properties. Perception, 36, 1368-1374. doi:10.1068/p5779 
Russell, R., Sinha, P., Biederman, I., \& Nederhouser, M. (2006). Is pigmentation important for face recognition? Evidence from contrast negation. Perception, 35, 749-759. doi:10.1068/p5490

Sadr, J., Jarudu, I., \& Sinha, P. (2003). The role of eyebrows in face recognition. Perception, 32, 285-293. doi:10.1068/p5027

Santos, I. M., \& Young, A. W. (2008). Effects of inversion and negation on social inferences from faces. Perception, 37, 1061-1078. doi:10.1068/ p5278

Sinha, P. (2002). Qualitative representations for recognition. Lecture Notes for Computer Science, 2525, 249-262.

Sinha, P., Balas, B. J., Ostrovsky, Y., \& Russell, R. (2006). Face recognition by humans: 19 results all computer vision researchers should know. Proceedings of the IEEE, 94, 1948-1962. doi:10.1109/JPROC .2006 .884093

Subramaniam, S., \& Biederman, I. (1997). Does contrast reversal affect object identification? Investigative Ophthalmology \& Visual Science, 38, 998.

Tanaka, J. W., \& Farah, M. J. (1993). Parts and wholes in face recognition. The Quarterly Journal of Experimental Psychology, 46A, 225-245.
Taubert, J., \& Alais, D. (2011). Identity aftereffects, but not composite effects, are contingent on contrast polarity. Perception, 40, 422-436. doi: $10.1068 / p 6874$

Vuong, Q. C., Peissig, J. J., Harrison, M. C., \& Tarr, M. J. (2005). The role of surface pigmentation for recognition revealed by contrast reversal in faces and Greebles. Vision Research, 45, 1213-1223. doi:10.1016/j .visres.2004.11.015

White, M. (2001). Effect of photographic negation on matching the expressions and identities of faces. Perception, 30, 969-981. doi:10.1068/ p3225

Young, A. W., Hellawell, D., \& Hay, D. C. (1987). Configurational information in face perception. Perception, 16, 747-759. doi:10.1068/ p160747

Received September 10, 2012 Revision received February 7, 2013

Accepted February 19, 2013

\section{Call for Nominations}

The Publications and Communications (P\&C) Board of the American Psychological Association has opened nominations for the editorships of History of Psychology; Journal of Family Psychology; Journal of Personality and Social Psychology: Personality Processes and Individual Differences; Psychological Assessment; Psychological Review; International Journal of Stress Management; and Personality Disorders: Theory, Research, and Treatment for the years 2016-2021. Wade Pickren, PhD, Nadine Kaslow, PhD, Laura King, PhD, Cecil Reynolds, PhD, John Anderson, PhD, Sharon Glazer, PhD, and Carl Lejuez, PhD, respectively, are the incumbent editors.

Candidates should be members of APA and should be available to start receiving manuscripts in early 2015 to prepare for issues published in 2016. Please note that the P\&C Board encourages participation by members of underrepresented groups in the publication process and would particularly welcome such nominees. Self-nominations are also encouraged.

Search chairs have been appointed as follows:

- History of Psychology, David Dunning, PhD

- Journal of Family Psychology, Patricia Bauer, PhD, and Suzanne Corkin, PhD

- JPSP: Personality Processes and Individual Differences, Jennifer Crocker, PhD

- Psychological Assessment, Norman Abeles, PhD

- Psychological Review, Neal Schmitt, PhD

- International Journal of Stress Management, Neal Schmitt, PhD

- Personality Disorders: Theory, Research, and Treatment, Kate Hays, PhD, and Jennifer Crocker, $\mathrm{PhD}$

Candidates should be nominated by accessing APA's EditorQuest site on the Web. Using your Web browser, go to http://editorquest.apa.org. On the Home menu on the left, find "Guests." Next, click on the link "Submit a Nomination," enter your nominee's information, and click "Submit."

Prepared statements of one page or less in support of a nominee can also be submitted by e-mail to Sarah Wiederkehr, P\&C Board Search Liaison, at swiederkehr@apa.org.

Deadline for accepting nominations is January 11, 2014, when reviews will begin. 